\title{
Prevalence of incidental breast cancer and precursor lesions in autopsy studies: a systematic review and meta-analysis
}

\author{
Elizabeth T. Thomas ${ }^{1}$, Chris Del Mar ${ }^{2}$, Paul Glasziou², Gordon Wright ${ }^{1}$, Alexandra Barratt ${ }^{3}$ and Katy J. L. Bell ${ }^{2,3^{*}}$
}

\begin{abstract}
Background: Autopsy studies demonstrate the prevalence pool of incidental breast cancer in the population, but estimates are uncertain due to small numbers in any primary study. We aimed to conduct a systematic review of autopsy studies to estimate the prevalence of incidental breast cancer and precursors.

Methods: Relevant articles were identified through searching PubMed and Embase from inception up to April 2016, and backward and forward citations. We included autopsy studies of women with no history of breast pathology, which included systematic histological examination of at least one breast, and which allowed calculation of the prevalence of incidental breast cancer or precursor lesions. Data were pooled using logistic regression models with random intercepts (non-linear mixed models).

Results: We included 13 studies from 1948 to 2010, contributing 2363 autopsies with 99 cases of incidental cancer or precursor lesions. More thorough histological examination ( $\geq 20$ histological sections) was a strong predictor of incidental in-situ cancer and atypical hyperplasia ( $O R=126.8$ and 21.3 respectively, $p<0.001)$, but not invasive cancer $(\mathrm{OR}=1 \cdot 1, p=0.75)$. The estimated mean prevalence of incidental cancer or precursor lesion was 19.5\% (0.85\% invasive cancer $+8.9 \%$ in-situ cancer $+9.8 \%$ atypical hyperplasia).

Conclusion: Our systematic review in ten countries over six decades found that incidental detection of cancer in situ and breast cancer precursors is common in women not known to have breast disease during life. The large prevalence pool of undetected cancer in-situ and atypical hyperplasia in these autopsy studies suggests screening programs should be cautious about introducing more sensitive tests that may increase detection of these lesions.
\end{abstract}

Keywords: Breast neoplasms, Mass screening, Early detection of cancer, Autopsy

\section{Background}

Breast cancer is common [1] and its incidence has been rising [2], largely from increased early detection of cancer through breast screening [3]. For a woman deciding whether or not to undergo mammography screening, the potential benefits of screening include averting the development of advanced breast cancer and possible premature death from breast cancer. However, these benefits must be considered alongside potential harms, one being the overdiagnosis and overtreatment of screen-detected cancers

\footnotetext{
* Correspondence: katy.bell@sydney.edu.au

${ }^{2}$ Centre for Research in Evidence-based Practice, Faculty of Health Sciences and Medicine, Bond University, Robina, QLD 4229, Australia

${ }^{3}$ Sydney School of Public Health, Sydney Medical School, Edward Ford Building

(A27), University of Sydney, Fisher Road, Sydney, NSW 2006, Australia

Full list of author information is available at the end of the article
}

that would otherwise never become apparent during the woman's lifetime. Cancer overdiagnosis, the diagnosis of cancers which never declare themselves during the patient's lifetime, may result from the detection of cancers which are very slowly progressive, non-progressive or even regressive, and may include overdiagnosis of both invasive cancer and in-situ cancer [4-13]. Pre-cancer overdiagnosis may also occur where there is detection of lesions such as atypical hyperplasia which either do not advance or do so only very slowly.

Autopsy studies may be used to estimate the size of the prevalence pool of incidental cancer (and pre-cancerous lesions) among people not known to have specific cancers during life. The prevalence pool of incidental prostate cancer, for example, has been estimated as $5 \%$ at age $<30$ years 
rising to $59 \%$ by age $>79$ years [14]; and incidental thyroid cancer as $5.7 \%$ overall, and $11.2 \%$ when the tissue is examined more intensively [15]. As such, autopsy studies can provide an indication of the potential for overdiagnosis of specific cancers if efforts to detect preclinical cancers and pre-cancers are made. The prevalence pool of incidental breast cancer was investigated in a systematic review of autopsy studies in 1997, which reported a median rate of 1.3\% of undiagnosed invasive breast cancer and $8.9 \%$ of undiagnosed ductal carcinoma in-situ (DCIS) [16]. Potential breast cancer overdiagnosis remains just as relevant now as then, if not more so. Changes to screening programmes to include the screening of older women ( $>70$ years of age), have resulted in increased numbers of women undergoing screening [17-21], and theoretically could increase overdiagnosis disproportionately [7, 22]. Moreover, increasingly sensitive diagnostic screening technologies have meant increased detection of a number of precursor lesions: atypical ductal and atypical lobular hyperplasia (ADH and ALH respectively) in addition to ductal, and lobular carcinoma in-situ (DCIS and LCIS respectively).

In this study we aimed to update the estimated size of the prevalence pool (reservoir) of incidental breast cancer and precursor lesions at autopsy, and identify factors that were associated with increased prevalence of these lesions.

\section{Methods}

\section{Protocol and registration}

The review protocol was not registered.

\section{Selection}

We included autopsy studies of adult women (>age 18 years) who had no history of pre-existing breast disease and which included a systematic histological examination of at least one breast. We excluded studies that did not report the women's age, or methodically examine the breast microscopically. The principal outcomes were rates of incidental breast cancer (invasive and in situ cancer), and precursor lesions (atypical hyperplasia) diagnosed on histopathology.

\section{Searching}

We searched Medline and Embase using the terms listed below, with no restrictions on year published, type of publication, or language (search terms created by a librarian). To identify further papers for inclusion in the review we ran forward citation searches and checked the references of all papers identified by the search for inclusion in the review. Finally, we repeated our original search to identify any additional papers published during the period of data collection.

\section{Search strategy (Medline)}

1 exp. Breast Neoplasms/

2 exp. Breast/pa [Pathology]
3 Breast Diseases/pa [Pathology]

4 ((breast or mammary) adj3 (neoplasm* or neoplasia* or tumour* or tumor" or cancer" or carcinoma* or adenocarcinoma" or malignan" or pre-malignan" or premalignan*)).tw.

5 or/1-4

6 Autopsy/

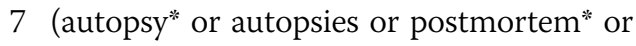
post-mortem" or post mortem*).tw.

86 or 7

95 and 8

\section{Validity assessment}

We planned a priori sub-group analyses for the following risk of bias study characteristics: consecutive versus nonconsecutive case selection, population based versus hospital based studies, and the possibility that breast cancer discovered at autopsy may have caused death. We also planned subgroup analysis of the following pathology validity characteristics: intensity of pathological examination (average number of sections submitted for histopathology per case); whether the histopathology reporting method used international standards (such as WHO [23]); and peer review of the histopathology diagnosis.

\section{Study selection and data abstraction}

Two authors (KB and ET) independently checked the titles and abstracts of all articles retrieved from Medline and Embase searches, and the full text was obtained if either author judged the article potentially relevant. The same two authors then independently checked all the full text articles for eligibility. Foreign language papers were translated into English using Google Translate. Disagreements were resolved through discussion with two further authors (CDM and PG).

Two authors independently extracted data for English papers (KB and $\mathrm{ET})$ and non-English papers (CDM and $\mathrm{KB}$ ) using standardized forms. We counted only one cancer or precursor lesion diagnosis per woman - in the case where more than one diagnosis was reported we chose the one of the highest grade/stage (i.e. invasive carcinoma > in situ carcinoma > atypical hyperplasia). We also only counted lesions which were not diagnosed during life - where this was uncertain, we were conservative and did not count the lesion. Disagreements were decided through discussion. We extracted data at the study level on: cancer and precursor prevalence, age, year that autopsies were performed, and the validity measures as described above. Where available, we also extracted data at the within-study level for women $<70$ years and $\geq 70$ years on: cancer and precursor prevalence.

\section{Quantitative data synthesis}

Our main summary measures were the prevalence of previously undiagnosed breast cancer or precursors: invasive 
breast cancer, in-situ breast cancer (DCIS and LCIS), and atypical hyperplasia (ADH and ALH). We pooled data from all studies using logistic regression models with random intercepts to represent the distribution of underlying cancer/precursor prevalences between different populations. This type of model also allowed for the nested structure of the data for the within-study analysis of age-specific prevalence estimates. We used the model to examine the impact of the validity characteristics on the prevalence estimates. SAS 9.4 was used for all analyses. The NLMIXED procedure was used to build the models, as has been recommended [24].

\section{Results}

We identified 1925 abstracts from Medline and Embase (search date 8th April 2016): 87 papers were retrieved for full text review; 71 of which did not meet our selection criteria (Fig. 1). Several of the remaining 16 studies used overlapping data: for each set of potentially overlapping reports, we chose the one that reported on the largest number of women, in the most detail (usually the most recent report), which resulted in 10 studies included from the original search. A further 10 potential papers were identified from the references and forward citation searches of the 10 included papers; of these 3 studies were included, with one [25] contributing two datasets (studies performed in two different countries). A total of 13 studies [25-37], contributing 14 datasets were included in the study level analysis, Table 1. There were 2363 women and 99 cases. There were separate data on cancer and precursor prevalence for women $<70$ years and $\geq 70$ years in 6 studies [26, $27,30,32,33,35]$ for the within-study analysis.

The median prevalence of: invasive cancer; in-situ cancer (DCIS and LCIS); atypical hyperplasia (ADH and ALH); and any of these lesions; were $1.1 \%$ (range $0-7.1 \%$; mean = $1.5 \%$ ), $0.0 \%$ (range $0-18.7 \%$; mean $=4.5 \%$ ), $3.4 \%$ (range 0 $14.5 \%$; mean $=4.8 \%)$ and $7.4 \% \quad(0-27 \%$; mean $=10.9 \%)$ respectively, Table 1 . The overall prevalence of incidental breast cancer or precursor lesion for the studies by the median year the autopsies were done (or year-of-publication if this information was unavailable) showed no visible trend in estimated prevalence over time, from the earliest study in 1947, to the most recent study in 2010, Fig. 2. We formally tested for temporal trend in the models below.

Predictors for incidental prevalence of each category (invasive cancer, in-situ cancer, atypical hyperplasia) and all categories combined, are presented in Table 2. Their prevalence differed between studies (test for random intercepts $p<0.001$ in each model). More thorough pathology examination yielded greater prevalences of in-situ cancer, atypical hyperplasia and all categories combined $(\mathrm{OR}=$ $126 \cdot 8,21.3$ and 29.3 , if $\geq 20$ sections taken on average compared to $<20$ sections taken on average respectively), but not invasive cancer $(\mathrm{OR}=1 \cdot 1)$, Fig. 3 . The prevalence of each category, and of all categories combined was not statistically higher for studies with an older average age of participants. There was weak evidence for higher prevalence of in-situ cancer in studies that used consecutive cases $(p=0.06)$, and of all categories combined for more recent studies $(p=0.09)$, but this disappeared after adjusting for thoroughness of pathology examination $(p=0.11$ and $p=0.37$ respectively). There was a higher prevalence of invasive cancer, (but not in-situ cancer or atypical hyperplasia), for the study (contributing two datasets) [25] reporting breast cancer discovered at autopsy which may have contributed to the women's deaths. None of the other available validity characteristics were statistically significant.

The mean prevalence of invasive cancer was $1.5 \%$. After excluding the study in which the breast cancer discovered at autopsy may have contributed to death [25], it was $0.85 \%$. The mean prevalence of in-situ cancer and atypical hyperplasia, with modelled adjustment upwards for less thorough studies, was $8.9 \%$ and $9.8 \%$ respectively. The overall mean cancer and precursor prevalence, with adjustment of in situ and precursor estimates upwards for less thorough studies, (no adjustment for invasive cancer), was $19 \cdot 5 \%(0.85 \%+8.9 \%+9 \cdot 8 \%)$.

When we repeated the analysis, limiting it to six studies with separate data on women $<70$ years and $\geq 70$ years, there were insufficient data to perform a direct withinstudy comparison while adjusting for thoroughness of pathology examination.

\section{Discussion}

Our systematic review of 13 studies (14 datasets) in ten countries over six decades of 2363 autopsies and 99 cases of incidental cancer or neoplastic precursor lesions, found that incidental breast cancer and its precursors are common in women not known to have breast disease during life. The estimates from these autopsy studies represent the best available evidence to answer the important question on the size of the prevalence pool of incidental breast cancer and precursor lesions.

The majority of incidental lesions appear to be precursors for invasive cancer (cancer in-situ and atypical hyperplasia). However the smaller prevalence pool of incidental invasive cancers appeared easier to find, with even the least thorough studies reporting cases. Unlike invasive cancer which was unrelated to the number of sections submitted for pathology examination, the estimated prevalence of cancer in-situ and atypical hyperplasia was strongly related to the thoroughness of microscopic examination. The odds of finding in-situ cancer and atypical hyperplasia were 127 times and 21 times higher respectively, in the studies where at least 20 sections were examined compared to those where less than 20 sections were examined. We could find no other clear predictors, including age, although this may be attributed to a paucity of data on women $\geq 70$ years. 


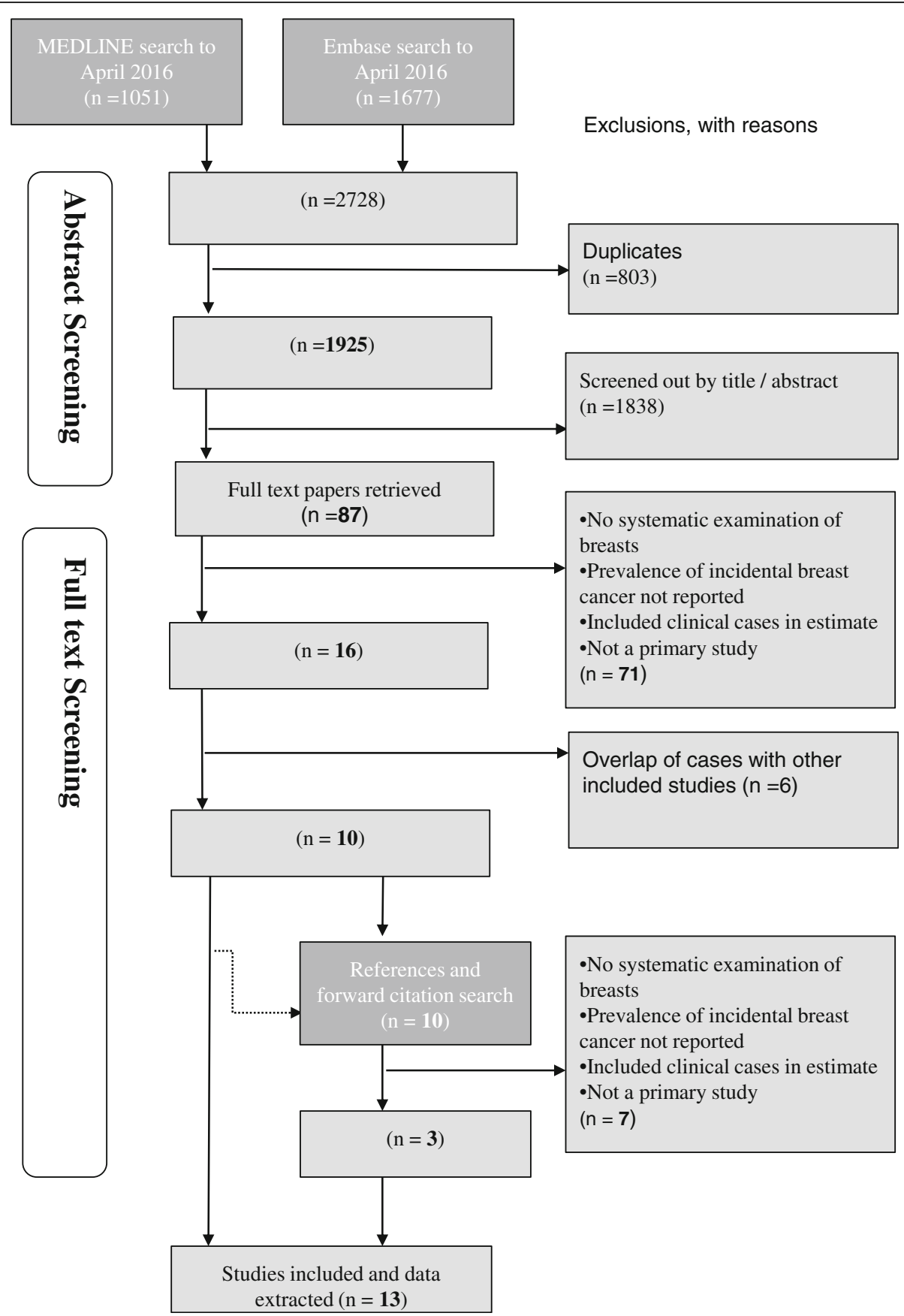

Fig. 1 Search and selection of primary studies for the meta-analysis

Our study builds on the evidence from a previous systematic review of incidental cancer discovered at autopsy [16]. We included six of the seven studies in that review (we excluded one study [38] as those data were included in a later report [33]). Our sensitive search strategy uncovered a further six reports which were published at the time of the previous review, but not discovered by them. We also found one more recent study [25] which contributed 2 datasets. Pooling these data enabled us to report on the substantial prevalence pool of invasive cancer, situ-cancer and precursor lesions (ADH and $\mathrm{ALH}$ ), and that the more thorough the microscopic examination, the more these lesions are discovered.

Autopsy rates are now much lower than previous decades; in addition, the widespread adoption of screening in many countries means that contemporary studies risk under-estimating the prevalence of incidental disease as much of this may have already been detected (and treated) 


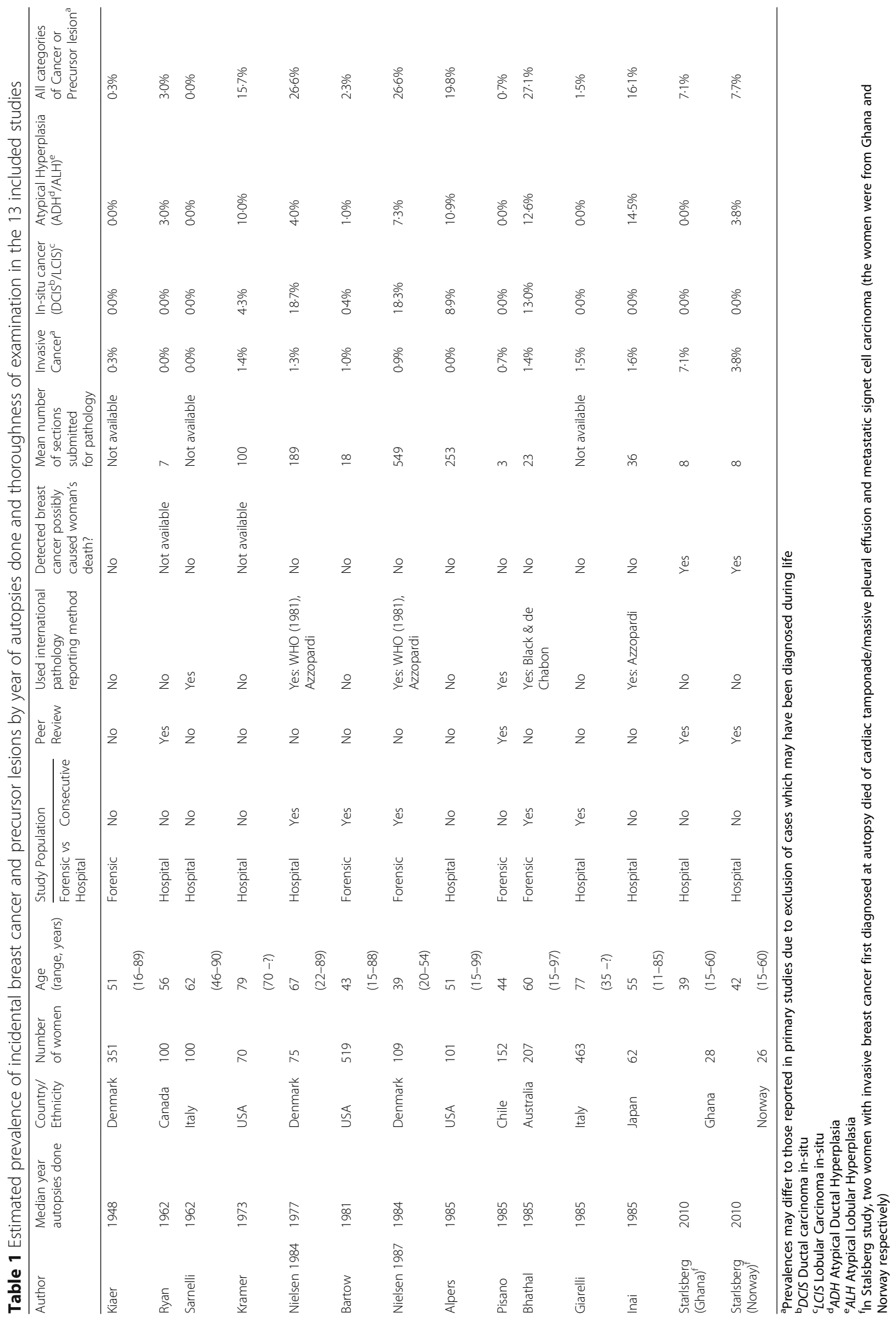



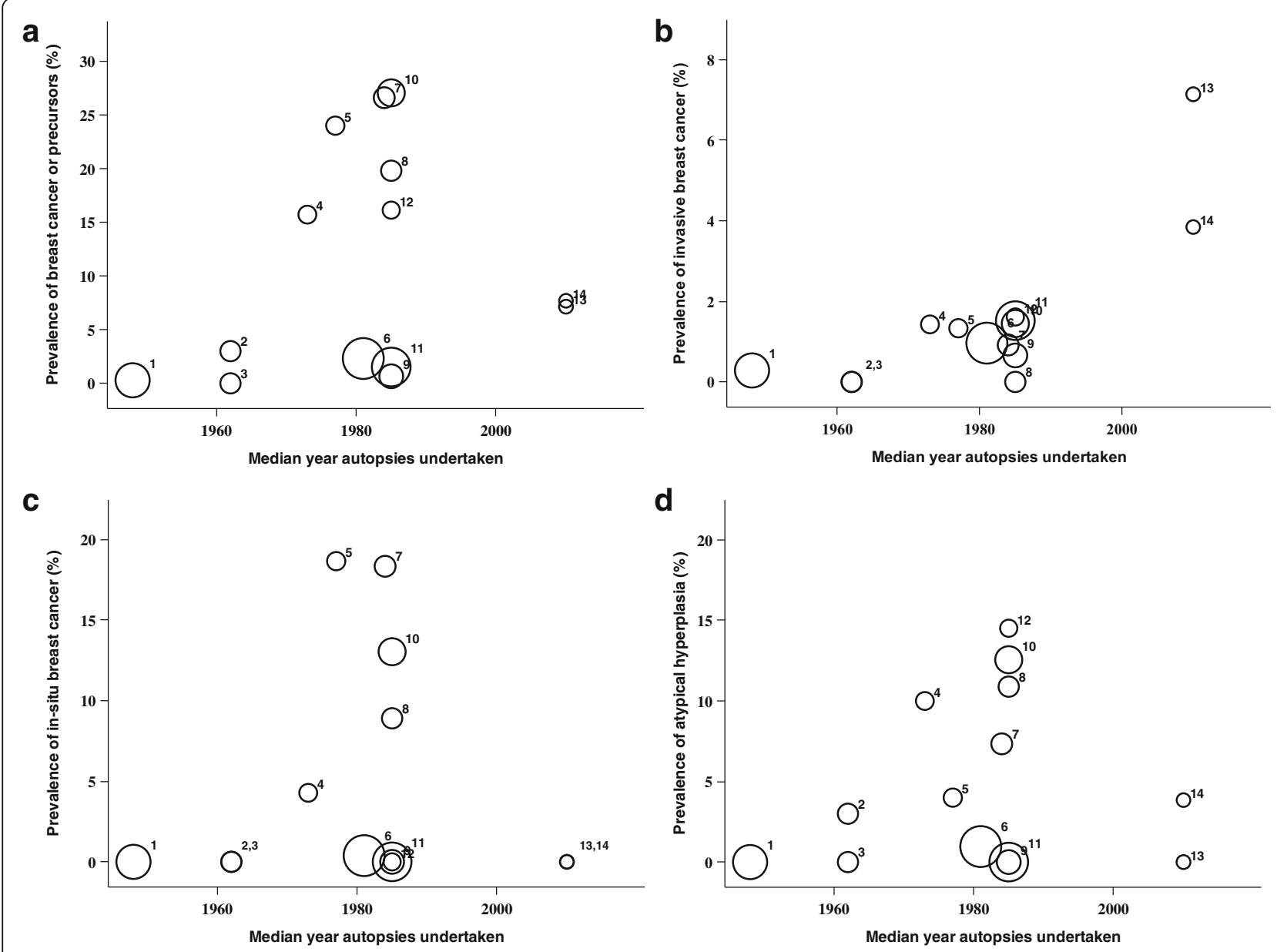

Fig. 2 Prevalence of incidental breast cancer or neoplastic precursor lesions in studies by median year of autopsies. $2 \mathbf{a}$ : Cancer or precursor lesion $2 \mathbf{b}$ Invasive cancer 2c: In-situ cancer 2d: Atypical Hyperplasia. Data points are proportional to total number of women in each study and are numbered according to year of study as follows: 1: Kiaer [26], 2: Ryan [27]; 3: Sarnelli [28]; 4: Kramer [29]; 5: Nielsen 1984 [30]; 6: Bartow [31]; 7: Nielsen 1987 [32]; 8: Alpers [33]; 9: Pisano [34]; 10: Bhathal [35]; 11: Giarelli [36]; 12: Inai [37]; 13: Stalsberg (Ghana) [25]; 14: Stalsberg (Norway) [25]

during life. Although the primary studies were conducted over a long time period (from 1948 to 2010), all but one were conducted in largely unscreened populations. The most thorough studies were conducted in the 1970s and 1980s, and included both hospital and forensic studies.

Limitations to this review include variation in the prevalence of incidental breast cancer and precursors across the studies, which could in part be due to underlying differences within the populations studied. Pathologists may have differing thresholds for classifying lesions [39] and differing levels of scrutiny with which they analyse lesions - which, as already discussed, was the strongest predictor of incidental breast cancer in-situ and atypical hyperplasia. Our review was also limited by the absence of data on the age-specific prevalence in most of the studies. We compared older and younger women's prevalences of incidental cancer and precursors but had insufficient data to make any conclusions on this. Insufficient information also prevented us from being able to compare cancer prevalence across race groups. In particular, there was a paucity of data related to women of African descent (only one study).

The size of the prevalence pool of incidental invasive breast cancer in unscreened populations may be used to provide an approximate lower bound for the extent of overdiagnosis associated with mammography screening: true overdiagnosis rates are likely to be at least this large. Our estimate of the prevalence of incidental invasive cancer, at $0.85 \%$, is much less than the current life-time prevalence of invasive cancer for women in the USA of $12.4 \%$ [40], or the life-time prevalence of screen-detected invasive cancer of $\sim 7.4 \%$ (assuming that about $60 \%$ of invasive cancers diagnosed during life are detected by mammography screening [41, 42]). If we assume that all of the prevalence pool of incidental cancer would be detected through screening (which is reasonable given the apparent ease with which incidental invasive cancer was detected in the autopsy studies), then the implications may be that at least $\sim 11 \% \quad(\sim 0 \cdot 85 / 7 \cdot 4 \%)$ of screen-detected invasive 


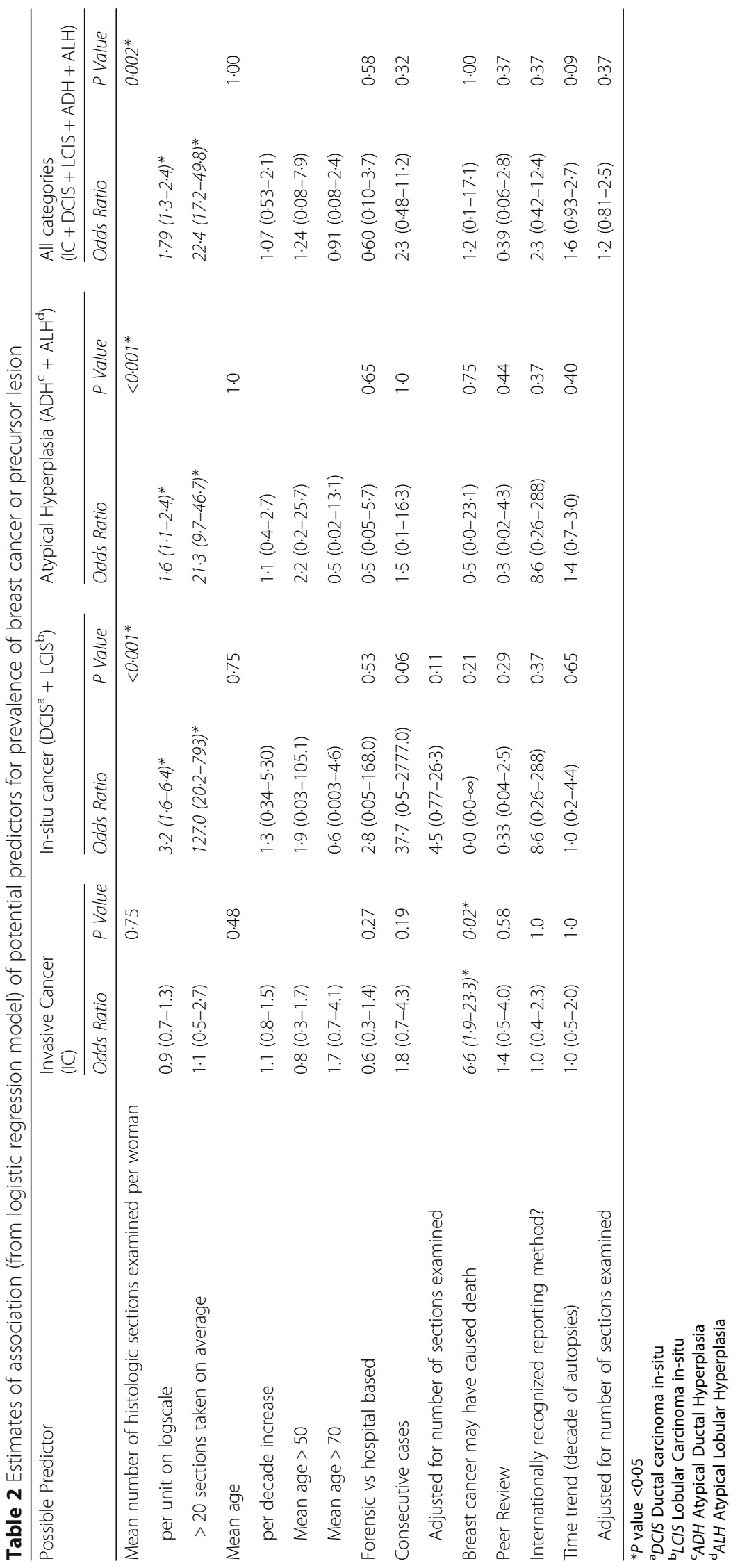




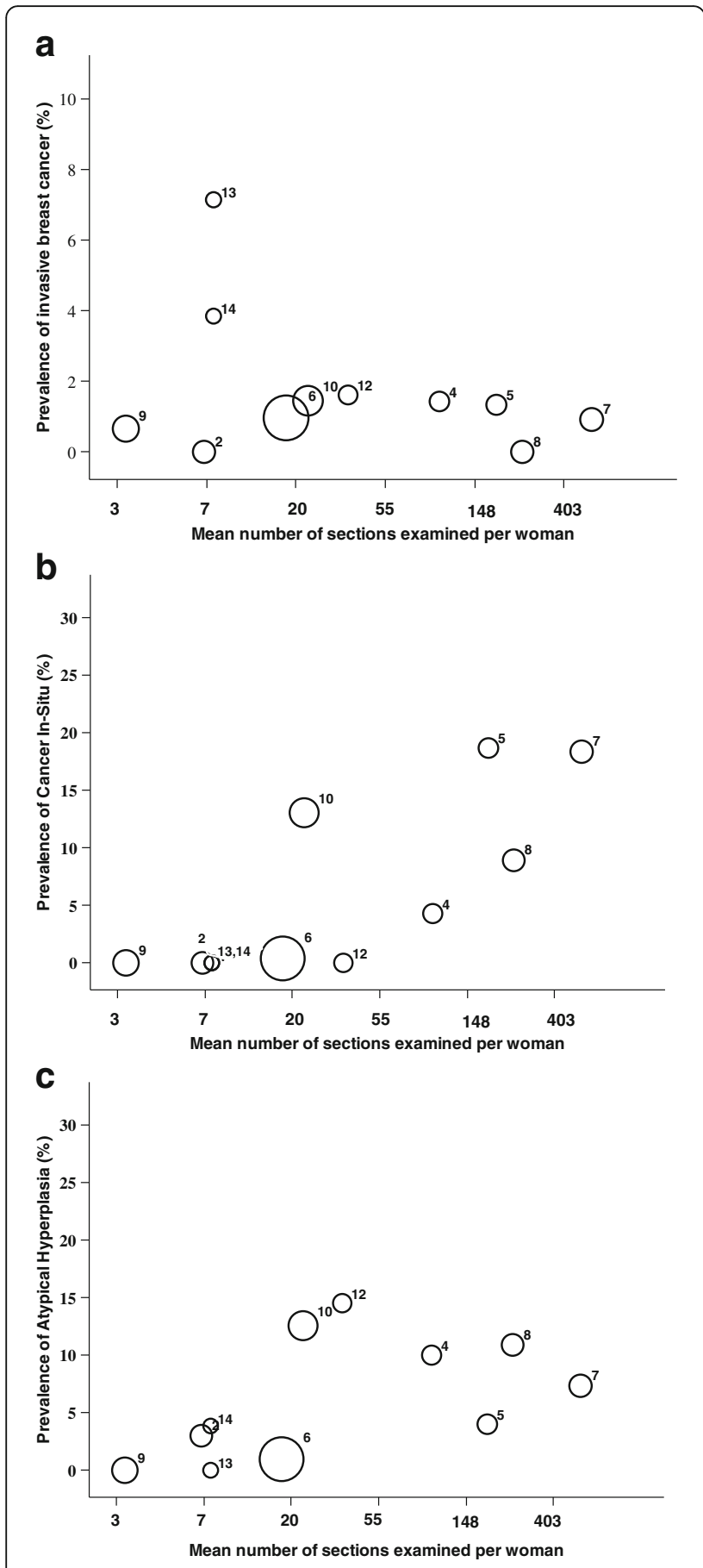

Fig. 3 Prevalence of incidental breast cancer or neoplastic precursor lesion in studies by thoroughness of pathology examination. 3a: Invasive cancer 3b: In-situ cancer 3c: Atypical Hyperplasia. Data points are proportional to total number of women in each study and are numbered according to year of study as follows: 1: Kiaer [26], 2: Ryan [27]; 3: Sarnelli [28]; 4: Kramer [29]; 5: Nielsen 1984 [30]; 6: Bartow [31]; 7: Nielsen 1987 [32]; 8: Alpers [33]; 9: Pisano [34]; 10: Bhathal [35]; 11: Giarelli [36]; 12: Inai [37]; 13: Stalsberg (Ghana) [25]; 14: Stalsberg (Norway) [25] cancers, or at least $\sim 7 \%(\sim 0.85 / 12.4)$ of all invasive cancers, are overdiagnosed. Invasive cancers that would have regressed if not detected by screening [43, 44] however, will cause the lifetime prevalence of overdiagnosed cancers to be greater than the incidental cancer prevalence discovered at autopsy in unscreened populations.

The excess lifetime prevalence of breast cancer in a regularly screened population may be used to provide an approximate upper bound for the extent of overdiagnosis associated with mammography screening: true overdiagnosis rates are likely to be no larger than this. The lifetime prevalence of invasive breast cancer in the USA in 19751977 (prior to the introduction of screening) was $9.4 \%$ (1 in 10.6 women). Since 1987 after roll-out of nation-wide mammography screening the life time prevalence has been stable at around $12.5 \%$ ( 1 in 8 women). Some of the increased risk in more recent times is because women are now less likely to die of other causes and because of changing risk factor levels, but the main explanation appears to be increased detection through mammography screening [45]. The expected decline in lifetime prevalence as screening rates stabilized has not eventuated [46] and a large proportion of the excess 3\% lifetime prevalence $(12.4 \%-9.4 \%)$, which has now persisted for 30 years, is likely to be due to overdiagnosis. The implications of this are that up to $\sim 40 \%(\sim 3 \% / 7.4 \%)$ of screen-detected invasive cancers, or up to $\sim 24 \%(\sim 3 / 12.4 \%)$ of all invasive cancers, may be currently overdiagnosed. Others' estimated overdiagnosis rates fall between our approximate lower and upper bounds [11, 43, 47-50].

For in-situ breast cancer, our estimate for the prevalence pool of incidental lesions is $\sim 9 \%$, much higher than the current life-time prevalence of $\sim 2.0 \%$ [40], and lifetime prevalence of screen-detected in situ cancer of 1.6\% (approximately $80-85 \%$ of in-situ cancers diagnosed during life are detected by screening [51]). This means there is a much higher probability of screen detected in-situ cancers being overdiagnosed (perhaps most are overdiagnosed), again consistent with estimates using other methods [11, 47].

The large pool of undetected cancer in-situ and atypical hyperplasia in these autopsy studies suggest caution for screening programs. First, as new breast screening technologies become more sensitive (e.g. digital mammography and breast tomosynthesis), it is likely that the proportion of overdiagnosed women will increase. Protocols for more intense biopsy sampling of screen detected abnormalities, or enhanced biopsy methods such as stereotactic vacuumassisted core biopsy, are also likely to further increase overdiagnosis rates. Accordingly, new technologies and biopsy protocols should evaluate whether any increased sensitivity is for clinically important or overdiagnosed cancers, for example by examining interval cancer rates in randomised comparisons of alternative screening technologies [52]. 
Second, expansion of mammography screening programs to include those aged $\geq 70$ years may also increase the risk of overdiagnosis and overtreatment [22]. The consequences of overtreating older women may also be more serious than for younger women because of their increased susceptibility to adverse effects of treatment [53].

\section{Conclusion}

This review has confirmed that there is a large prevalence pool of incidental breast cancer and precursor lesions present across all ages. Policy-makers, researchers, clinicians and women invited to undergo screening need to be aware of the extent of overdiagnosis so breast cancer detection can be improved and women do not undergo treatment for an inconsequential finding detected through breast cancer screening.

\section{Abbreviations}

ADH: atypical ductal hyperplasia; ALH: atypical lobular hyperplasia; DCIS: ductal carcinoma in-situ; LCIS: lobular carcinoma in-situ; OR: Odds Ratio; USA: United States of America; WHO: World Health Organisation

\section{Acknowledgements}

We thank Sarah Thorning and Justin Clark for their help with the literature search.

\section{Funding}

The authors have received funding from the Australian National Health and Medical Research Council (Early Career Fellowship No. 1013390, Australia Fellowship No. 527500 and Program Grant No 633003). The funders had no role in design and conduct of the study; collection, management, analysis, and interpretation of the data; and preparation, review, or approval of the manuscript.

\section{Availability of data and materials}

All data generated or analysed during this study are included in this published article and its supplementary information files.

\section{Authors' contributions \\ ETT is a medical student at Bond University. She developed the ideas, acquired and interpreted data and edited the paper. CDM is Professor of Public Health at Bond University and a part-time General Practitioner. He developed the ideas, interpreted data and edited the paper. PG is Director of the Centre for Research in Evidence Based Practice at Bond University and a part-time General Practitioner. He developed the ideas, interpreted data and edited the paper. GW is Professor of Pathology at Bond University. He developed the ideas, interpreted data and edited the paper. AB is Professor of Public Health at the University of Sydney. She developed the ideas, interpreted data and edited the paper. KLB is a Senior Research Fellow at the University of Sydney and Bond University. She conceived and developed the ideas, acquired data, did the analysis, interpreted data, wrote the first draft, edited the paper and is guarantor. All authors have read and approved the final version of the manuscript and agreed to be fully accountable for ensuring the integrity and accuracy of the work.}

\section{Ethics approval and consent to participate}

Not applicable.

\section{Consent for publication}

Not applicable.

\section{Competing interests}

All authors have completed the ICMJE uniform disclosure form at http:// www.icmje.org/coi_disclosure.pdf and declare: no support from any organisation for the submitted work; no financial relationships with any organisations that might have an interest in the submitted work in the previous three years, no other relationships or activities that could appear to have influenced the submitted work.

\section{Publisher's Note}

Springer Nature remains neutral with regard to jurisdictional claims in published maps and institutional affiliations.

\section{Author details}

${ }^{1}$ Faculty of Health Sciences and Medicine, Bond University, Robina, QLD 4229, Australia. ${ }^{2}$ Centre for Research in Evidence-based Practice, Faculty of Health Sciences and Medicine, Bond University, Robina, QLD 4229, Australia. ${ }^{3}$ Sydney School of Public Health, Sydney Medical School, Edward Ford Building (A27), University of Sydney, Fisher Road, Sydney, NSW 2006, Australia.

Received: 5 June 2017 Accepted: 21 November 2017

Published online: 02 December 2017

\section{References}

1. World Health Organization: Breast cancer: prevention and control. http:// www.who.int/cancer/detection/breastcancer/en/index1.html (2016). Accessed 23 Apr 2016

2. Ferlay J, Soerjomataram I, Ervik M et al. GLOBOCAN 2012 v1.0: Cancer Incidence and Mortality Worldwide In: IARC CancerBase No.11. International Agency for Research on Cancer, Lyons. 2013. http://globocan.iarc.fr/. Accessed 24 Apr 2016

3. Welch HG, Black WC. Overdiagnosis in cancer. J Natl Cancer Inst. 2010;102: 605-13.

4. Bleyer A, Welch HG. Effect of three decades of screening mammography on breast-cancer incidence. N Engl J Med. 2012;367:1998-2005.

5. Esserman $L$, Thompson IM, Reid B, Nelson P, Ransohoff DF, Welch HG, et al. Addressing overdiagnosis and overtreatment in cancer: a prescription for change. Lancet Oncol. 2014;15:e234-42.

6. Marmot MG, Altman DG, Cameron DA, Dewar JA, Thompson SG, Wilcox M. The benefits and harms of breast cancer screening: an independent review. Br J Cancer. 2013;08:2205-40.

7. Barratt A. Overdiagnosis in mammography screening: a 45 year journey from shadowy idea to acknowledged reality. BMJ. 2015;350:h867.

8. Carter J, Coletti R, Harris R. Quantifying and monitoring overdiagnosis in cancer screening: a systematic review of methods. BMJ. 2015;350:7773.

9. Etzioni R, Gulati R, Mallinger L, Mandelblatt J. Influence of study features and methods on Overdiagnosis estimates in breast and prostate cancer screening. Ann Intern Med. 2013:58:831-8.

10. Nelson H, Pappas M, Cantor A, Griffin J, Daeges M, Humphrey L. Harms of breast cancer screening: systematic review to update the 2009 U.S. preventive services task force recommendation. Ann Intern Med. 2016;164:256-67.

11. Ripping TM, Verbeek AL, Fracheboud J, de Koning HJ, van Ravesteyn NT, Broeders MJ. Overdiagnosis by mammographic screening for breast cancer studied in birth cohorts in The Netherlands. Int J Cancer. 2015;137:921-9.

12. Puliti D, Duffy S, Miccinesi G, De Koning H, Lynge E, Zappa M, et al. Overdiagnosis in mammographic screening for breast cancer in Europe: a literature review. J Med Screen. 2012:19:42.

13. Brawley OW. Accepting the existence of breast cancer Overdiagnosis. Ann Intern Med. 2017:166:364-5.

14. Bell KJ, Del Mar C, Wright G, Dickinson J, Glasziou P. Prevalence of incidental prostate cancer: a systematic review of autopsy studies. Int J Cancer. 2015; 137:1749-57.

15. Furuya-Kanamori L, Bell KJ, Clark J, Glasziou P. SA. Prevalence of differentiated thyroid cancer in autopsy studies over six decades: a metaanalysis. J Clin Oncol. 2016;34:3672-86.

16. Welch HG, Black WC. Using autopsy series to estimate the disease "reservoir" for ductal carcinoma in situ of the breast: how much more breast cancer can we find? Ann Intern Med. 1997:127:1023-8.

17. Health and Social Care Information Centre, Screening and Immunisations team.Breast Screening Programme, England 2011-2012. In: Health and Social Care Information Centre 2013. http://www.hscic.gov.uk/catalogue/ PUB10339/bres-scre-prog-eng-2011-12-rep.pdf. Accessed 24 Apr 2016.

18. Jacklyn G, Howard K, Irwig L, Houssami N, Hersch J, Barratt A. Impact of extending screening mammography to older women information to support informed choices. Int J Cancer. 2017; doi:10.1002/ijc.30858.

19. International Cancer Screening Network. Breast cancer screening programs in 26 ICSN countries, 2012: organization, policies and program reach. In: National Cancer Institute. 2016. https://healthcaredelivery.cancer.gov/icsn/ breast/screening.html. Accessed 27 Aug 2017. 
20. Altobelli $E$, Lattanzi A. Breast cancer in EuropeanUnion: an update of screening programmes as of march 2014 (review). Int J Oncol. 2014;45:1785-92.

21. Cancer CPA: Organized Breast Cancer Screening Programs in Canada: Report on Program Performance in 2007 and 2008. In Canadian Partnership Against Cancer; Public Health Agency of Canada 2013. http://www.phacaspc.gc.ca/publicat/2008/obcsp-podcs-03-04/pdf/obcsp-podcs-03-04-eng. pdf. Accessed 28 Aug 2017.

22. de Glas NA, de Craen AJM, Bastiaannet E, Op 't Land EG, Kiderlen M, van de Water W, et al. Effect of implementation of the mass breast cancer screening programme in older women in the Netherlands: population based study. BMJ. 2014; doi:10.1136/bmj.g5410.

23. Sobin L. Histological typing of breast Tumours, 2nd edn. Geneva: world health Organization; 1981.

24. Hamza TH, van Houwelingen HC, Stijnen T. The binomial distribution of meta-analysis was preferred to model within-study variability. J Clin Epidemiol. 2008;61:41-51.

25. Stalsberg $\mathrm{H}$, Adjei EK, Owusu-Afriyie O. No difference in the prevalence of benign breast changes between women from Ghana and Norway: an autopsy study. Breast Cancer Res Treat. 2015;51:177-82.

26. Kiaer W. Relationship of fibroadenomatosis ("chronicmastitis") to cancer of the breast. M.D. thesis. Copenhagen: University of Copenhagen; 1954

27. Ryan JA, Coady CJ. Intraductal epithelial proliferation in the human breast-a comparative study. Can J Surg. 1962:5:12-9.

28. Sarnelli R, Squartini F. Fibrocystic condition and "at risk" lesions in asymptomatic breasts: a morphologic study of postmenopausal women. Clin Exp Obstet Gynecol. 1991;18:271-9.

29. Kramer WM, Rush BF. Mammary duct proliferation in the elderly. A histopathologic study. Cancer. 1973;31:130-7.

30. Nielsen M, Jensen J, Andersen J. Precancerous and cancerous breast lesions during lifetime and at autopsy. A study of 83 women. Cancer. 1984;54:612-5.

31. Bartow SA, Pathak DR, Black WC, Key CR, Teaf SR. Prevalence of benign, atypical, and malignant breast lesions in populations at different risk for breast cancer. A forensic autopsy study. Cancer. 1987;60:2751-60.

32. Nielsen M, Thomsen $J$, Primdahl S, Dyreborg U, Andersen JA. Breast cancer and atypia among young and middle-aged women: a study of 110 medicolegal autopsies. Br J Cancer. 1987;56:814-9.

33. Alpers CE, Wellings SR. The prevalence of carcinoma in situ in normal and cancer-associated breasts. Hum Pathol. 1985;16:796-807.

34. Pisano R, Campodonico I, Valdivia I, Norambuena L, Duran V, Pisano R, Retamales I. Morphological study of 152 breasts from medico-legal autopsies. Rev Chil Obstet Ginecol. 1986;51:42-55.

35. Bhathal PS, Brown RW, Lesueur GC, Russell IS. Frequency of benign and malignant breast lesions in 207 consecutive autopsies in Australian women. Br J Cancer. 1985;51:271-8.

36. Giarelli L, Stanta G, Delendi M, Sasco AJ, Ribolli E. Prevalence of female breast cancer observed in 517 unselected necropsies. Lancet. 1986;2:864

37. Inai K, Yamamoto A, Arihiro K, Khatun N, Kurihara K, Takeda S. Epithelial hyperplasia of the breast. Comparison of incidence between cases of breast carcinoma and control autopsy specimens with immunohistochemical observation of blood group antigens. Acta Pathol Jpn. 1992;42:193-200.

38. Wellings SR, Jensen HM, Marcum RG. An atlas of subgross pathology of the human breast with special reference to possible precancerous lesions. J Natl Cancer Inst. 1975;55:231-73.

39. Elmore JG, Longton GM, Carney PA, Geller BM, Onega T, Tosteson AN, et al. Diagnostic concordance among pathologists interpreting breast biopsy specimens. JAMA. 2015;313:1122-32.

40. Cancer Statistics SEER. Review (CSR) 1975-2014. In: surveillance, epidemiology, and end results. Program. 2017; https://seer.cancer.gov/csr/ 1975_2014/. Accessed 27 Aug 2017

41. Glasziou P, Houssami N. The evidence base for breast cancer screening. Prev Med. 2011:53:100-2.

42. Breen $\mathrm{N}$, Yabroff $\mathrm{K}$, Meissner $\mathrm{H}$. What proportion of breast cancers are detected by mammography in the United States? Cancer Detect Prev. 2007;31:220-4.

43. Zahl PH, Maehlen J, Welch HG. The natural history of invasive breast cancers detected by screening mammography. Arch Intern Med. 2008;168:2311-6.

44. Zahl PH, Gotzsche PC, Maehlen J. Natural history of breast cancers detected in the Swedish mammography screening programme: a cohort study. Lancet Oncol. 2011;12:1118-24.

45. Feuer $E$, Wun $L$, Boring C, Flanders $W$, Timmel $M$, Tong $T$. The lifetime risk of developing breast cancer. J Natl Cancer Inst. 1993;85:892-7.
46. Kessler L, Feuer E, ML B. Projections Of the breast cancer burden to U.S. women: 1990-2000. Prev Med. 1991;20:170-82

47. Baines CJ, To T, Miller AB. Revised estimates of overdiagnosis from the Canadian National Breast Screening Study. Prev Med. 2016:90:66-71.

48. Morrell S, Barratt A, Irwig L, Howard K, Biesheuvel C, Armstrong B. Estimates of overdiagnosis of invasive breast cancer associated with screening mammography. Cancer Causes Control. 2010;21:275-82.

49. Zahl PH, Strand BH, Maehlen J. Incidence of breast cancer in Norway and Sweden during introduction of nationwide screening: prospective cohort study. BMJ. 2004;328:921-4.

50. Kalager M, Adami HO, Bretthauer M, Tamimi RM. Overdiagnosis of invasive breast cancer due to mammography screening: results from the Norwegian screening program. Ann Intern Med. 2012;156:491-9.

51. Kerlikowske K. Epidemiology of ductal carcinoma in situ. JNCI Monographs. 2010;2010:139-41.

52. Bell K, Bossuyt P, Glasziou P, Irwig L. Assessment of changes to screening programmes: why randomisation is important. BMJ. 2015; doi:10.1136/bmj. h1566.

53. de Glas NA, Kiderlen M, Bastiaannet E, de Craen AJ, van de Water W, van de Velde CJ. Postoperative complications and survival of elderly breast cancer patients: a FOCUS study analysis. Breast Cancer Res Treat. 2013;138:561-9.

\section{Submit your next manuscript to BioMed Central and we will help you at every step:}

- We accept pre-submission inquiries

- Our selector tool helps you to find the most relevant journal

- We provide round the clock customer support

- Convenient online submission

- Thorough peer review

- Inclusion in PubMed and all major indexing services

- Maximum visibility for your research

Submit your manuscript at www.biomedcentral.com/submit
) Biomed Central 